\title{
THE EVOLUTION OF QUASARS AND THEIR CLUSTERING
}

\author{
P.S. OSMER \\ Astronomy Department, The Ohio State University
}

\section{Introduction}

George Djorgovski's message to me on behalf of the organizing committee suggested that I discuss 1) Evolution of the Quasar Luminosity Function; 2) Possible Differences among Radio-Loud, Radio-Quiet, and X-Ray Selected Samples; 3) Quasars as Probes of Clustering and Large-Scale Structure at High Redshift; 4) Searches for Extremely Distant Quasars; 5) Theoretical Implications; 6) Major Surveys in Progress and Planned; and 7) What Surveys Should We Do in the Future. To cover all these topics in a 25minute talk and 7-page article is a challenge, but I am happy to try.

As we approach the end of the century and indeed the millennium, it has been fashionable to write books and articles about the end of subjects such as history and science. What about the end of quasars? That is a more legitimate question, because we have not extended the high-redshift limit at $z=4.9$ since 1991 and there is quantitative evidence that the space density is falling steeply for $z>4$. However, in reviewing the literature, I can assure you that we have not reached the end of publications nor work in the field. The ADS abstract service lists 498 articles on quasars in 1995 alone. The number of known quasars is of order 10,000 and will grow by another order of magnitude with planned surveys. Advances in technology now enable surveys for quasars over large fractions of the entire sky to significantly fainter flux limits than could be reached before at X-ray, optical, IR, and radio wavelengths. Furthermore, there is the new and very positive trend of making the data sets from such surveys publicly available, in many cases as soon as the data can be processed. I predict that such public access will greatly increase the scientific results from the surveys by enabling more studies of the data on a broader range of topics than any 
single person or group could accomplish, to say nothing of permitting the cross-checking of results, which is a basic part of our science.

The plan of this talk is to cover in the sections below the questions posed in the first paragraph (except that question 2 will be discussed only briefly in $\S 6)$.

\section{Evolution of the Quasar Luminosity Function}

In discussing the evolution of quasars, we should remember that the fundamental physical question is how quasars themselves form and evolve. However, what we derive from observations is the quasar luminosity function (QLF). And, while we have made progress in characterizing how the QLF evolves with redshift, I do not think we have yet established that the parameterization of the QLF as showing luminosity evolution, for example, means that the quasars themselves are undergoing luminosity evolution in the sense of a long-lived population of objects that are bright at $z \approx 2$ and then fade toward the present day. Rather, I think that establishing how quasars evolve physically should be one of the main goals of future surveys. Boyle (1991, Figure 2) gives a nice description of the observational issues on this matter.

A second important point is understanding what it takes to determine the evolution of the QLF, namely, large, calibrated, quantitative surveys whose detection efficiency is well characterized (see Hewett \& Foltz 1994 for an excellent discussion of this topic). Suppose, for example, that we were to require 10 quasars per magnitude bin and 10 magnitude bins per redshift over 10 redshift intervals. This would mean 1,000 quasars overall. The challenge in assembling such a sample, which we have yet to do, is not the total number, but filling the bins at the extremes in redshift and absolute magnitude. High-luminosity quasars are rare, as are quasars at $z>4$, and one must cover large areas of the sky. Low-luminosity quasars are much more common, but their apparent magnitudes become increasingly faint with increasing redshift. No single survey to date has provided all the data we need. However, we have been able to make progress with relatively small samples because the evolution of the QLF is so pronounced: the space density of quasars increases by a factor of 1000 between redshifts 0 and 2 .

At present, determination of the QLF for $z<2.2$ is based primarily on the Durham/AAT UVX survey (Boyle 1991) and the Large Bright Quasar Survey (Hewett, Foltz, and Chaffee 1993). For $z>3$, there are three calibrated surveys (Warren, Hewett, \& Osmer 1994 [WHO], Schmidt, Schneider, \& Gunn 1995 [SSG], and Kennefick, Djorgovski, \& de Carvalho 1995 [KDC]). The KDC results provide confirmation that the observed space density of quasars at $z>4$ does decline as indicated by the earlier work 
of WHO and SSG. A linear plot of space density against lookback time shows a remarkable peak when the universe was approximately $15 \%$ of its present age. This striking feature suggests that quasar activity was very concentrated at redshifts near 2 to 3 .

Of course there still are challenges and questions for this picture. Have bright quasars with $z>4$ been missed (Irwin, McMahon, \& Hazard 1991 [IMH])? Are significant numbers of quasars obscured by dust (Webster et al. 1995)? Are there other significant populations of quasars that have been missed by surveys to date, such as optically quiet quasars or ones found only by variability (e.g., Hawkins \& Veron 1995)? While I agree that some classes of quasars are missed by optical surveys based on color and emission lines, I am not yet persuaded that the overall picture of quasar evolution needs to be changed. For example, Boyle and di Matteo (1995) argue from a ROSAT-selected sample of quasars that a significant number of quasars is not being missed. But, this is a scientific question that is resolvable with current technology. Quantitative surveys that follow the criteria of Hewett \& Foltz (1994) can provide the answers.

\section{Quasars as Probes of Clustering \& Large-Scale Structure at High Redshifts}

Because of their great luminosity, quasars are in principle the best tracers of clustering and large-scale $(1000 \mathrm{Mpc})$ structure at $z>1$. But, they are rare objects, which presumably originated in high-density peaks in the primordial fluctuations, and they yield sparse sampling of large-scale structure. Shanks \& Boyle (1994) give a good account of previous work in the field and the current state of the subject. Using a compilation of available surveys, they present convincing evidence that quasar clustering on scales up to $10 h^{-1} \mathrm{Mpc}$ is detected at the $4 \sigma$ level of significance and furthermore argue in favor of stable clustering for both quasars and galaxies out to $z \approx 2$.

The situation at larger scales has been hampered by lack of surveys over sufficiently large areas on the sky, but there have been discoveries of supercluster-sized groups of quasars. It has been difficult to establish the statistical significance of such groups because their sizes have been comparable to the surveys in which they were found. Most recently, Graham, Clowes, \& Campusano (1995) apply a minimum spanning-tree analysis to the data and conclude that the several $\sim 100 h^{-1} \mathrm{Mpc}$ groups found by Crampton et al.(1989) and themselves are real.

Looking ahead, this is a subject that the Sloan Digital Sky Survey and the $2 \mathrm{dF}$ quasar survey should definitely settle. With large, connected areas of up to $10,000 \mathrm{deg}^{2}$ and sample sizes up to 100,000 objects, we can expect 
an improvement of the $S / N$ ratio for clustering of more than a factor of 10 at scales up to $10 h^{-1} \mathrm{Mpc}$. At the $100 h^{-1} \mathrm{Mpc}$ scales we will finally have adequate control fields to establish the nature of the supercluster-sized groups.

\section{Searches for Extremely Distant Quasars $(z>5)$}

The results of WHO, SSG, KDC, and IMH indicate that the expected number of quasars with $z>5$ is small. Future efforts will need a combination of area on the sky and faint limiting magnitude. Good sensitivity at $\lambda>0.8 \mu \mathrm{m}$ will also be required because Lyman $\alpha$ absorption along the line of sight will make quasars faint at shorter wavelengths.

The Sloan Digital Sky Survey is expected to make a very important contribution because of the large area on the sky it will cover. At present several groups are making targeted searches for extremely distant quasars. Kennefick and Smith are leading a survey that makes use of the second Palomar Survey in combination with z-band $(0.9 \mu \mathrm{m})$ images from the Burrell and Curtis Schmidts at NOAO to cover $2000 \mathrm{deg}^{2}$. Irwin \& McMahon and Hewett \& Warren are two of the groups in the U.K. making use of the La Palma Observatory and U.K. Schmidt plates to search for $z>5$ quasars.

Current expectations, based on the searches for $z>4$ quasars to date and the QLFs derived by WHO, SSG, and KDC, for example, indicate that at $I=18 \mathrm{mag}$, more than $1000 \mathrm{deg}^{2}$ will probably be needed to find one quasar at $z>4.8$. At $\mathrm{I}=20$, the limit of the Sloan Survey, one quasar is expected in 15 to $300 \mathrm{deg}^{2}$ (SSG to WHO form of the decline with increasing redshift). Thus, the Sloan Survey, with its planned coverage of $10,000 \mathrm{deg}^{2}$ has an excellent chance of finding a significant number of quasars with $z>5$.

\section{What Do the Observations Tell Us about the Origins of $\mathrm{Nu}$ - clear Activity? About the Formation of Large Scale Structure and Galaxies?}

The strong peak in the space density of quasars near redshift 2-3 begs the question, 'Are we seeing the epoch of quasar formation?' Recall that in the standard model for quasars, the observed luminosity is a function of the black-hole mass and the fueling rate of material being accreted. Thus, it is very likely that the peak in space density is related to galaxy formation and interactions at high redshift. Furthermore, if quasars represent high-density peaks in structure formation, then the clustering and largescale distribution of quasars should yield valuable information for models of structure formation. 
There has been considerable theoretical work on these topics (see, for example, the article by Haehnelt and Rees (1993) and the series by Loeb and collaborators (e.g., Loeb \& Rasio 1994). Katz et al. (1994) have performed numerical simulations in a biased cold dark matter model that include gas dynamics. Their results (see their striking color illustrations) give a good demonstration that numerous dense sites, suitable for the formation of quasars, can occur at $z=4.5$ and even at $z=8$ in models where galaxies form later, showing that the ubiquitous CDM model continues to meet the challenges put to it.

I think we can expect a very fruitful interplay of theory and observation in this area in the next few years because of the rapid advances in theory and computation on the one hand and forthcoming observational results from radio interferometry, X-ray observations, and Hubble Space Telescope, for example.

\section{What Do We Expect to Learn from On-going and Planned} Surveys Such as SDSS, POSS-II, ROSAT, and Special Surveys?

Let me begin with some of the large on-going surveys. The second Palomar Survey (POSS-II or DPOSS), which is described by Djorgovski (this volume, p. 424), will be just as fundamental and important to our field as POSS-I was.

The FIRST survey (Becker et al. 1995) is making use of the VLA to cover the area planned for the Sloan Digital Sky Survey (see below) in the North Galactic Polar Cap. The goal is to map 1 million radio sources to a 1 mJy flux limit with positional accuracies of $<1$ arcsec to eliminate ambiguity in subsequent source identifications. The data are being made available to the public as soon as they are ready. Among the survey objectives for quasars are the determination of the evolution of radio-loud quasars, for which FIRST will provide a definitive sample of objects. FIRST has already yielded 51 relatively bright radio quasars that were previously unknown. Furthermore, its flux limit is so sensitive that even "radio-quiet" quasars are being found ${ }^{1}$.

ROSAT has enabled significant progress on several quasar programs. Bade et al. (1995) have found 269 quasars and AGNs from ground-based followup observations of the ROSAT All-Sky Survey. Boyle et al. (1994) confirmed 107 quasars with $0<z<3.2$ with their optical spectroscopy of very deep pointed observations and estimated that quasars and AGNs contribute about 0.4 of the X-ray background. From the finding of a type

\footnotetext{
${ }^{1}$ Obviously radio-quiet quasars are not radio silent; rather, their radio luminosity is too low to have been detected in previous surveys. They do constitute a different class of quasars from the radio-loud objects.
} 
2 quasar in a deep ROSAT exposure, Almaini et al. (1995) noted that such sources might account for the elusive hard component of the X-ray background. Comastri et al. (1995) have carried out a detailed study of available ROSAT and other X-ray data to model the different components of the X-ray background. They conclude that quasars and AGNs can account for $74 \%$ of the background. Thus, we can see that ROSAT has made significant contributions to X-ray studies of quasars.

With regard to the X-ray properties of quasars and AGNs, Schartel et al. (1996) analyzed ROSAT data and provided confirmation for the Einstein result that radio-quiet quasars have steeper spectra than do radioloud quasars. In addition, the spectrum becomes flatter with increasing redshift, presumably because of the emission from higher energies being shifted into the observed energy band.

The ROSAT All-Sky Survey Bright-Source Catalog is now publically available and can be accessed via the World Wide Web.

Turning to new, large surveys that will soon begin, consider the Sloan Digital Sky Survey (SDSS, Gunn \& Weinberg 1995). It is planned to cover $10,000 \mathrm{deg}^{2}$ in the northern Galactic polar cap, plus strips of sky in the southern galactic hemisphere. Direct imaging observations will be made in $u g r i z$ filters. The goal for quasar research is to identify and obtain redshifts for 100,000 quasars to 20 th magnitude. SDSS is expected to yield tremendous improvement in our knowledge of 1) the QLF and its evolution for a wide range in redshift; 2) the space density of quasars with $z>5 ; 3$ ) the nature and evolution of quasar clustering and large-scale structure; 4) rare types of quasars and bright quasars suitable for absorption-line studies at high spectral resolution.

A complementary and also very important survey is the $2 \mathrm{dF}$, which is described by Smith (this volume, p. 348). Boyle \& Smith and Croom \& Shanks have used UK Schmidt exposures in $U$ and $B$ filters to find UVX quasar candidates in two strips of the sky that can be covered with the $2^{\circ}$ field multi-object spectrograph of the Anglo-Australian telescope. The goal is to have a sample 30,000 quasars to $\sim 21$ st magnitude that will be well suited for studies of quasar clustering and large-scale structure for redshifts up to $\sim 2.2$.

\section{What Surveys Should We Contemplate in the Future?}

Because of the tremendous progress we can expect from surveys like the ones mentioned in the previous section, I am tempted to repeat my old joke that soon we are going to need a bigger sky for future work. However, I would be wrong - we can already identify important key surveys that either are already feasible or will soon be in the near future. 
In X-rays, we have only been able to reach faint quasars/AGNs or very high-redshift quasars that are now routinely observable at optical wavelengths with extremely long ( $>10^{5} \mathrm{sec}$ ) exposures with ROSAT. AXAF will provide a significant improvement in sensitivity and energy coverage and should clearly be used in conjunction with $10-\mathrm{m}$ class telescopes for the optical and spectroscopic identification of faint X-ray sources. Objectives will be to settle the outstanding questions posed by the X-ray background and its unusual spectral energy distribution and to see if important classes of quasars/AGNs have been missed in other surveys and at other wavelengths. $A X A F$ should also make equally important contributions to our physical understanding of the nature of quasars because $\mathrm{X}$-rays probe the inner-most observable regions of quasars.

At radio wavelengths, optical and spectroscopic identification of the faint sources from surveys like FIRST will be key to understanding the nature, formation, and evolution of radio-loud and radio-quiet quasars.

Searches for the highest $z$ quasars will be needed to understand their epoch of formation, chemical abundances (and consequences for chemical evolution), and the reddening and absorption along the line of sight. The advent of multiple, large-format CCD cameras on 4-m class telescopes will enable surveys for such quasars to considerably fainter limits than SDSS over tens and possibly hundreds of square degrees on the sky.

Results in the last year from the Hubble Deep Field and spectroscopy with Keck indicate that we will be able to establish the relationship of quasars and AGNs to their host galaxies and their environments out to high redshift. This should yield significant new insights on the formation and evolution of both quasars/AGNs and galaxies.

The use of large-format IR array detectors on large telescopes and future IR work from space will be key to settling the questions raised about the number of quasars/AGNs that are hidden by dust. It has not been possible to date to achieve the needed combination of faint limiting sensitivity and large enough area on the sky, but we are on the verge of significant advances in our capabilities. As with X-rays, the astrophysical studies of quasars with IR observations will be very important. The optical spectral features on which much of our knowledge of quasar/AGN emission-line regions is based are shifted to the IR in the $z>4$ objects.

Finally, continued advances in detectors and telescopes will enable us to attack clustering and large scale structure problems not covered by $2 \mathrm{dF}$ and SDSS, in particular those requiring large samples of very faint quasars.

Thus, if we are approaching any end of quasars, it is the first, exploratory phase of their properties and evolution. Where we really are is at the beginning of developing an understanding of their true nature and how they form and evolve. 


\section{Acknowledgements}

I am grateful to the Scientific Organizing Committee for the opportunity to speak at the meeting, to G. Hasinger for updating me on ROSAT results, and to B. Peterson for reviewing a draft of the article.

\section{References}

Almaini, O., Boyle, B.J., Griffiths, R.E., Shanks, T., Stewart, G.C., \& Georgantopoulos, I. 1995, Mon.Not.R.astron.Soc., 277, L31

Bade, N., Fink, H.H., Engels, D., Voges, W., Hagen, H.-J., Wisotzki, L., and Reimers, D. 1995, Astron.Astrophys.Suppl., 110, 469

Becker, R.H., White, R.L., \& Helfand, D.J. 1995, Astrophys.J., 450, 559

Boyle, B.J. 1991, in Ann. NY Acad. Sci 647, Texas/ESO-CERN Symposium on Relativistic Astrophysics, Cosmology, and Fundamental Physics, ed. J.D. Barrow et al., 14

Boyle, B.J., Shanks, T., Georgantopoulos, I., Stewart, G.C., \& Griffiths, R. E. 1994, Mon.Not.R.astron.Soc., 271, 639

Boyle, B.J., \& di Matteo, T. 1995, Mon.Not.R.astron.Soc., 277, L63

Comastri, A., Setti, G., Zamorani, G., \& Hasinger, G. 1995, Astron.Astrophys., 296, 1

Crampton. D., Cowley, A.P., \& Hartwick, F.D.A. 1989, Astrophys.J., 345, 59

Graham, M.J., Clowes, R.G., \& Campusano, L.E. 1995, Mon.Not.R.astron.Soc., 275, 790

Gunn, J., \& Weinberg, D. 1995, in "Wide Field Spectroscopy and the Distant Universe," ed. S.J. Maddox \& A. Aragòn-Salamanca (Singapore: World Scientific), 3

Haehnelt, M.G., \& Rees, M.J. 1993, Mon.Not.R.astron.Soc., 263, 168

Hawkins, M.R.S., \& Veron, P. 1995, Mon.Not.R.astron.Soc., 275, 1102

Hewett, P.C., \& Foltz, C.B. 1994, Publ.Astron.Soc.Pacific, 106, 113

Hewett, P.C., Foltz, C.B., \& Chaffee, F.H. 1993, Astrophys.J., 406, L43

Irwin, M., McMahon, R.G., \& Hazard, C. 1991, in "The Space Distribution of Quasars," ed. D. Crampton, Astron.Soc.Pacific Conference Series, 21, 117

Kennefick, J.D., Djorgovski, S.G., \& de Carvalho, R.R 1995, Astron.J., 110, 2553

Katz, N., Quinn, T., Bertschinger, D., and \& Gelb, J.M. 1994, Mon.Not.R.astron.Soc., 270, L71

Loeb, A., \& Rasio, F.A. 1994, Astrophys.J., 432, L52

Schartel, N., Walter, R., Fink, H.H., \& Trumper, J. 1996, Astron.Astrophys., 307, 33

Schmidt, M., Schneider, D.P., \& Gunn, J.E. 1995, Astron.J., 110, 68

Shanks, T., \& Boyle, B.J. 1994 Mon.Not.R.astron.Soc., 271, 753

Warren, S.J., Hewett, P.C. \& Osmer, P.S. 1994, Astrophys.J., 421, 412

Webster, R.L., Francis, P.J., Peterson, B.A., Drinkwater, M.J., \& Masci, F.J. 1995, Nature, 375, 469 